\title{
Shape Boundary Tracking with Hidden Markov Models
}

\author{
Terry Caelli \\ Department of Computing Science \\ Research Institute for Multimedia Systems (RIMS) \\ The University of Alberta, Edmonton, Alberta, CANADA T6G 2E9 \\ tcaelli@ualberta.ca
}

\begin{abstract}
This paper considers a Hidden Markov Model (HMM) for shape boundary generating which can be trained to be consistent with human expert performance on such tasks. That is, shapes are defined by sequences of "shape states" each of which has a probability distribution of expected image features (feature "symbols"). The tracking procedure uses a generalization of the Viterbi method by replacing its "best-first" search by "beam-search" so allowing the procedure to consider less likely features as well in the search for optimal state sequences. Results point to the benefits of such systems as an aide for experts in depiction shape boundaries as is required, for example, in Cartography.
\end{abstract}

Keywords: Hidden Markov Models, symbolic descriptions of boundaries, predicting human performance, Viterbi Search.

\section{Introduction}

Though generating the boundary or shape of single objects seems quite simple, there are still no automated procedures which can reliably perform such tasks. On closer inspection of aerial images, for example, it can be seen that the local variabilities of color/intensity which experts use to infer features are difficult to encode by machines without additional knowledge including characteristics of human performance. This paper deals with this latter perspective and explores how Hidden Markov Models can be applied to the generation of symbolic descriptions of low-level image features such as shape boundaries in ways which are consistent with specific task demands and image types.

The proposed model defines structures in terms of sequences of "shape states" and the proposed HMM generates such states through a model based upon: (1) defining shape boundaries as sequences of (boundary) "Shape States" (SS) that determine the local boundary structures at given positions and associated directions for interpolation between such positions and states; (2) image feature extraction; (3) image feature registration as a discrete set of feature types: "feature symbols"; (4) learning to bind such symbols with Shape States; (5) the use of constrained search over neighboring image regions to find the appropriate feature symbols to evidence Shape States which, in turn, generate 
(predict) the shape boundary. In the following sections a brief overview of these components is provided.

\section{$2 \quad$ Feature Extraction and Shape States}

For shape (boundary) encoding, it is necessary to encode feature values which represent intensity/color contrast, sidedness, orientation and related properties of the boundary. In this project we have used multi-scaled oriented approximations to what we term "petal filters", a variation of the "wedge filters" [7] where each "petal" pair is defined about a center position, $\boldsymbol{x}$, by:

$$
\left\{G_{i}\left(\boldsymbol{x}+\boldsymbol{u}_{\boldsymbol{i}}, \operatorname{cov}_{i}\right), G_{i}\left(\boldsymbol{x}-\boldsymbol{u}_{\boldsymbol{i}}, \operatorname{cov}_{i}\right)\right\}
$$

as shown in Figure 1. Here, $n$ corresponds to the number of oriented filters, $\boldsymbol{x}$ to the center position of the filter, $\boldsymbol{u}$ to the offset for the gaussian $\left(G_{i}\right)$ center, and $\operatorname{cov}_{i}$ to the modulation covariance defining the weighting of pixel values over the filter region. We choose such pairs of offset gaussians to represent the sensitivity to orientation information as a non-monotonic function of distance from the center, with the nearer distances and larger distances having less sensitivity due to resolution and integration window limits respectively. Specific configurations of such filters form the feature "symbol" codes (feature symbols: FS) which are indexed via the Shape States in the HMM (see Figure 2).

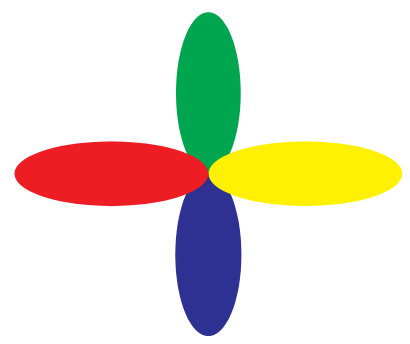

Fig. 1. Petal Filters are defined by linear combinations of symmetrically offset gaussians (even) in a set of orientations tuned by sets of covariance functions. A variety of image properties can be directly encoded by this filter such as corners, orientation and, in general, color contrast. Specific types of petal spatio-chromatic configurations ("flowers") form the fundamental feature symbol encoders in the current system.

Here 8 basic shape states (SS) were used corresponding to inside and outside corners over vertical (Figure 2) and oblique orientations. These SSs are used to define our domain shape models as composed of right-angle polygons - a form of particular relevance to Geographic Information System (GIS) data formats. 
a
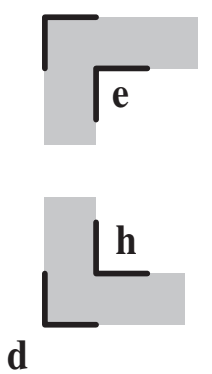
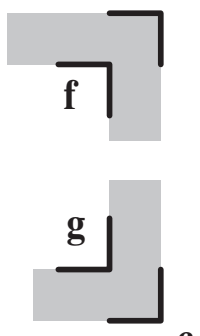

C

Fig. 2. 8 shape states (SS) used to symbolically describe shape boundaries in terms of: (1) SS sequences- defined by their individual and transition probabilities; (2) the probabilities of the feature types (see Figure 1) given each SS - all defined in the W-HMM

For this reason the proposed model is termed the "What-HMM" (W-HMM) as positional information is not explicitly encoded in the mode1.

\section{Generating Symbolic Descriptions}

In recent years most feature tracking models have been developed to model environmental exploration and feature detection in the area of robot navigation and models for visual "attention" in humans with particular interest in integrating peripheral and foveal vision[6]. Combining multi-scaled filtering, Kalman filtering(predictive mode), Hidden Markov Models and, in general, adaptive control models are typical of what has been used 511. However, this type of approach has not been used for basic tasks like shape boundary recognition and production.

More formally, the boundary tracking W-HMM is defined as follow 2 . Let: $T=$ length of the sequence of observed features (symbols); $N=$ number of Shape States (SS); $M=$ number of feature types - feature symbols (FS); $\Omega_{X}=q_{1}, \ldots q_{N}$ : the underlying SS sequence defining the shape; $\Omega_{O}=v_{1}, \ldots, v_{M}$ : the observed FS sequence; $X_{t}$ : random variable denoting the SS at time t; $O_{t}$ : random variable denoting the observed $\mathrm{FS}$ at time $\mathrm{t} ; \sigma=o_{1}, \ldots, o_{T}$ : the sequence of observed feature symbols. The HMM probabilities are then defined by:

${ }^{1}$ Although defining shapes only in terms of expected SS transitions implies an equivalence class of shapes invariant to the distances between SS's, such SS's are evidenced from feature types which, from the training data, constrain the types of expected positional ranges for such shapes.

2 The following formulation is adapted from http://ftp.cs.brown.edu/research/ai/dynamics/tutorial/Documents /HiddenMarkovModels.html 
$A=a_{i j}$ such that $a_{i j}=\operatorname{Pr}\left(X_{t+1}=q_{j} \mid X_{t}=q_{i}\right)$ : the SS transition probabilities.

$B=b_{i}$ such that $b_{i}(k)=\operatorname{Pr}\left(O_{t}=v_{k} \mid X_{t}=q_{i}(t)\right)$ : the state-conditional FS probabilities. ties.

$\pi=\pi_{i}$ such that $\pi_{i}=\operatorname{Pr}\left(X_{0}=q_{i}\right)$ : defines the initial or prior SS probabili-

The $W$-HMM for generating the shape boundary is defined by the five-tuple $\left(\Omega_{X}, \Omega_{O}, A, B, \pi\right)$. We let $\lambda=\{A, B, \pi\}$ denote the parameters for a given $W$ $H M M$ with fixed $\Omega_{X}$ and $\Omega_{O}$.

This model is motivated by (but essentially different from) earlier work of Rimey and Brown [5] who proposed WHAT and WHERE systems for the control of attention. In their "Augmented HMM" system (AHMM) feedback was introduced to allow a WHERE-HMM to re-initialize new positions from the detection of, for example, features in the peripheral field of view. The states of their HMM corresponded to position movement types and the symbols to actual movements and their associated probabilities. The W-HMM differs from this in a number of ways. One, the underlying states correspond to Shape States (for example, the 8 states defined in Figure 2) and the W-HMM encodes the relationships between Shape States (SS) and observed filter response types. This type of HMM has interesting properties which, by definition, do not involve the explicit encoding of positional information but, rather, what features to look for within a specified spatial range ("scale") extracted from, for example, movement distance statistics used during training or by constrained neighborhood search. It allows for a more general definition of "shape" in so far as it permits the occurrences of symbols in more positions that what are delimited by HMMs which explicitly encode positional information. However this W-HMM ("What-HMM") does require search strategies which, themselves, could be incorporated into the W-HMM or explicitly encoded by an additional HMM - an extension not examined here.

\subsection{Generating HMM Inputs and Initial Estimates}

A Fuzzy version of the K-Means algorithm [2] has been used to determine the predominant types of features (the petal filter outputs are defined by a vector of "petal" values corresponding to the similarity of spatial and chromatic values at each petal orientation - see Figure 1a).

Initial estimates for each object's HMM model $(\lambda)$ probabilities are determined from human performance statistics during training involving: (1) the observed SS relative frequencies; (2) the observed relative frequencies of shape state (SS) transitions between consecutive states; (3) the state conditional FS relative frequencies in the observed training data. The Baum-Welsh algorithm was then used to update the model estimates from the observed sequence. This is a form of Expectation-Maximization where the current model parameters and the observed sequence are combined to determined new weighted parameter estimates. Following this, a new version of the Viterbi procedure[3] - "Viterbi Search" - is 
(a)

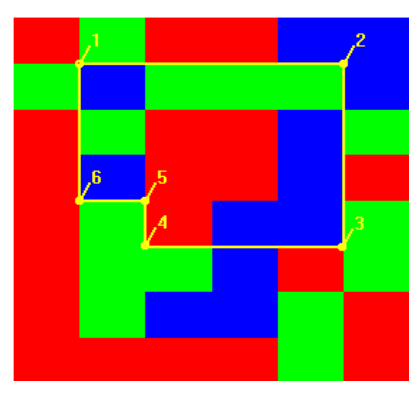

(b)

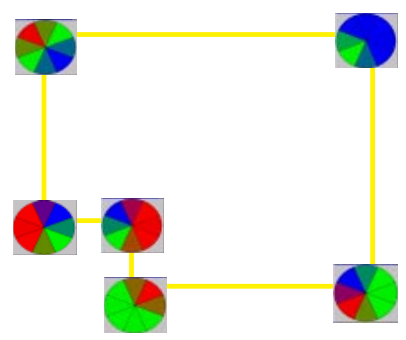

(c)

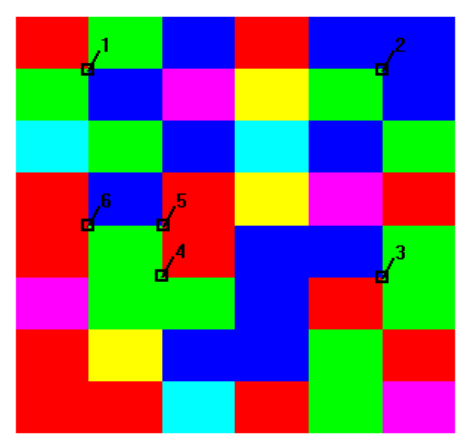

Fig. 3. (a) Shows input shape. (b) Shows extracted feature cluster centroids (Feature States: FS) associated with the corner with petal filter outputs nearest to the cluster (FS). Note how these clusters reflect the major types of FS characteristics of the shape boundary. (c) Resultant shape tracking via the W-HMM algorithm.

used to determine the degree to which each HMM can predict SS sequences from observed flower (FS) sequences.

\subsection{Viterbi Search}

The standard Viterbi algorithm is a best-first search method for finding the most likely state sequences which matches the observed symbol sequnece[3]. The two basic problems with the method are that, being best-first, it is not optimal search neither allowing for back-tracking nor for a queue of possible combinations of states and symbols (SS and FS) beyond the most likely pair. The latter limitation is particularly relevant in sequence production where the most likely symbol may not be observed - but, for example, the second most likely is - so allowing the 
W-HMM to continue propagating a predicted state sequence, given the existence of specific feature types in the predicted range of positions in the image.

A more general "Viterbi Search" algorithm has been used to overcome these limitations. Here, at each time, a queue is constructed from the list of possible SSs and FSs and ordered by the products of SS and FS probabilities. This queue is "popped" until an FS is detected so selecting the combination of SS and FS at that time.

Accordingly, for the W-HMM at RunTime, given that the system or expert has selected a feature symbol (FS) at a given initial position $\left(t_{0}\right)$, it must search for the predicted new state(s) within the search window which is defined by the pixel corridor, in this case a $+/-5$ pixels range, about the line formed between the current and predicted SS directions. The search direction is determined from the orientations of the current $\mathrm{SS}(\mathrm{t})$ and predicted $\mathrm{SS}(\mathrm{t}+1)$. In all, then, the search is initiated along such paths and candidate positions are selected as a function of the most likely feature symbol (top of queue). At a given $\mathrm{SS}(\mathrm{t})$ this process searches the queue until the most likely feature $(\mathrm{p}(\mathrm{SS}(\mathrm{t}+1) / \mathrm{SS}(\mathrm{t})) * \mathrm{p}(\mathrm{FS}(\mathrm{t}+1) / \mathrm{SS}(\mathrm{t}+1)))$ can be instantiated through the occurrence of a predicted feature at the candidate position.

\subsection{Assessing HMM Performance}

The Viterbi algorithm defines the most likely state sequence in terms of the final (joint) maximum probability of the state sequences given the observed symbol sequence. This measure is neither optimal (as the Viterbi algorithm only corresponds to best-first search and not optimal search) nor sensitive enough (the probability value is typically very small and determined from the products of a large number of probabilities) to capture the degree to which the derived state sequence is likely to generate the specific observation sequence. For these reasons we have developed an additional method for assessing the result of the Viterbi search and, in turn, the degree to which the Baum-Welch procedure produces a model representative of the observed symbol sequences. This method is based upon computing the Hamming distance between observed and predicted observation sequences using a MonteCarlo method. This is, for a given model we generate sequences by randomly selecting states, state transition and observations according to the model probabilities. This is computed a number of times to result in a mean and standard deviation of distances between observed $(O(t))$ and predicted $(P(i))$ observation sequences of length $T$. We have used a normalized Hamming distance defined by:

$$
d(P, O)=\sum_{i=1}^{T} \Phi(P(i), O(i)) / T
$$

where

$$
\Phi(P(i), O(i))=\left\{\begin{array}{l}
0 \text { if } P(i) \equiv O(i) \\
1 \text { otherwise }
\end{array}\right.
$$


This results in a direct measure of the likelihood than the particular observed sequence would match what can be predicted from the model without any optimal search for the "best state sequence" and acts as a baseline to compare with the Viterbi solution. The measure can also be interpreted as a simple Edit Distance in so far as it indexes the number of edits required to transform the predicted into the observed symbol sequence.

The Viterbi procedure results in an estimate of the best state sequence. This state sequence can then be used to generate observation sequences using MonteCarlo methods to sample the symbols according to their conditional probability densities - resulting in the "constrained" MonteCarlo measure of distance between predicted and observed symbol sequence. Comparing these values indicates the "gain" in using Viterbi. In the following examples we have used this latter procedure and measure to select and fit the model to observations.

\section{Illustrations and Experimental Results}

Our current experiment involved the detection, recognition and tracking of shape boundaries in three different types of images - shapes whose boundaries are defined by specific color contrasts, shapes embedded in additive gaussian noise and remotely sensed groups of houses. In each case the task is restricted to the detection of ordered sequences of right-angle corners from the petal filter outputs.

We first illustrate how the W-HMM functions with recognition of shape corners defined by specific combinations of colours. Figure 3(a) shows an initial training image where the system is given examples of corner types (labeled corners). Figure 3(b) shows the six resultant cluster centroids and the corners which are closest to these values in feature space - mapped as oriented segments of a circle. Figure 3(c) shows how the Viterbi search procedure finds the shape boundary embedded in a quite different montage of color squares although difficult to detect by the human eye. This merely illustrates the core concept behind the W-HMM: that shape is encoded by the dependencies between specific types of shape states as evidenced from the detection of sequences of specific feature types.

In the second experiment we focus more on the degree to which the feature extraction and Viterbi search methods are robust enough to activate the correct state sequences even when the shapes are embedded in a significant amount of noise. The W-HMM was trained on 7 different patterns (Figure 4(Top Left)) and tested on unseen patterns with various degrees of zero mean additive gaussian noise (Figure 4). The model estimation procedures discussed in Sections 2 and 3 were used and, in this case they were able to perfectly generate the training sequences $(d(P, O)=0)$. Results with unseen shapes embedded in the most extreme noise case ( $\sigma=31.8$ for each color using an 24-bit color image format) are shown in Figure 4. In this case performance the Hamming distance between observed and predicted was $d(P, O)=0.08$, or, $8 \%$ of the SSs were incorrectly labeled. 

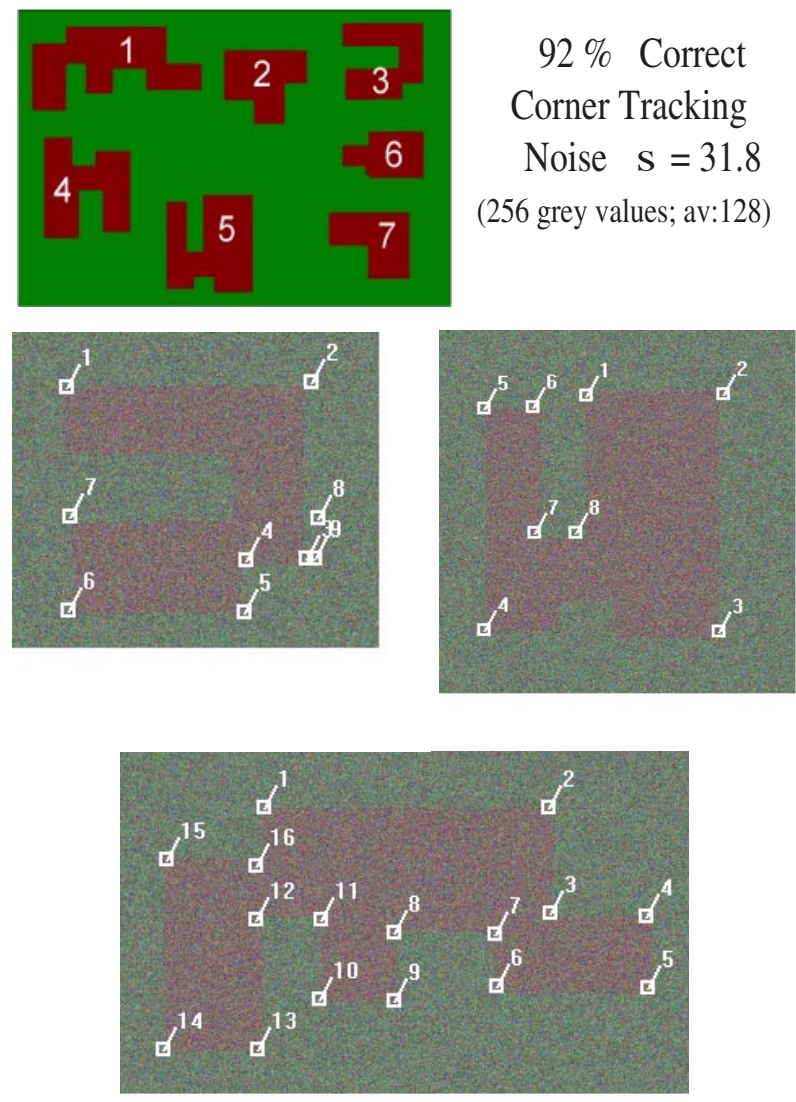

Fig. 4. Shows performance on unseen patterns using additive gaussian noise.

Another example of the W-HMM involved remotely sensed images of buildings. In this case we used six different images and building types - all being rectangular polygons - as illustrated in Figure 5. Again, the same model estimation procedures were used on the training data. Testing on these training data resulted in $d(P, O)=0$. On unseen buildings from three new images 57 out of a total of 68 corners were correctly tracked (Figure 5).

These results point to the general feasibility of the W-HMM as an aide to experts when tracking shape boundaries is required since in all these experiments the expected number of edits on predicted SSs was less than 10\%. All errors occurred with features which could not activate the most likely combination of symbol type and state transition and with Viterbi queues with more uniform probability densities. This is to be expected and it also follows that the more objective ways to define a given HMM lies in the mutual information of the A and B matrices and the "second-order" or self-correcting property. 
(a)

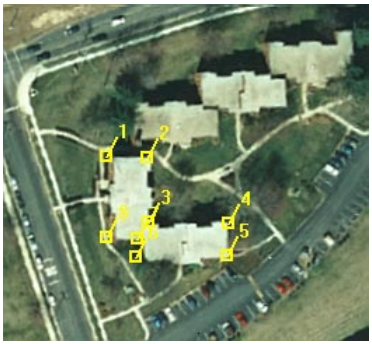

(d)

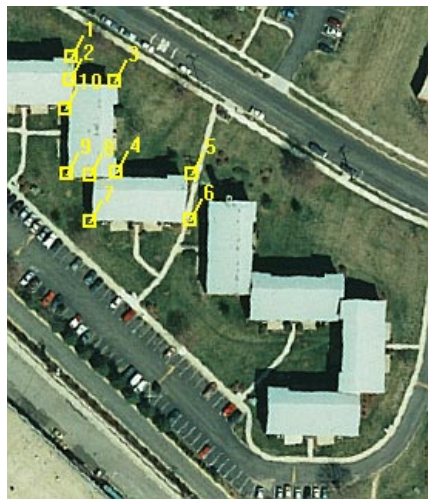

(e)

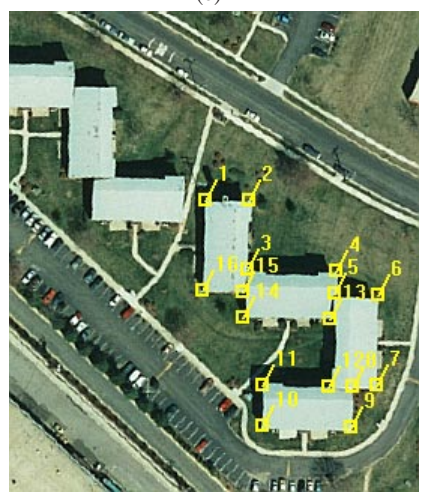

(b)
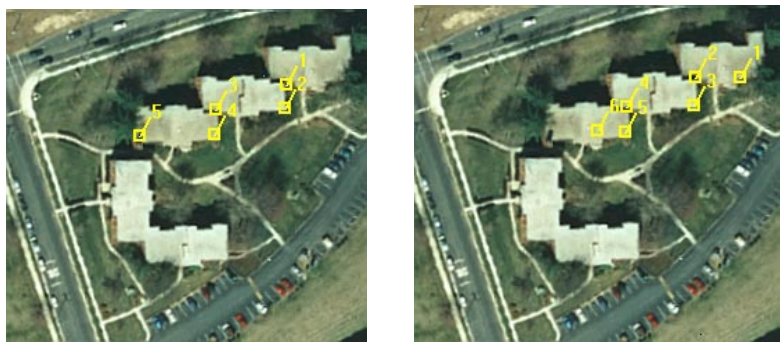

(f)

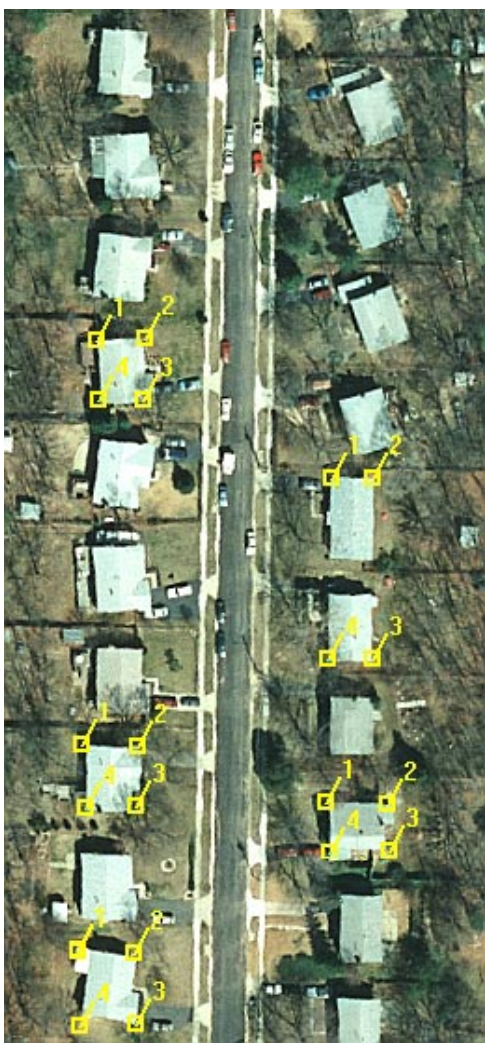

Fig. 5. Shows examples of tracking performance on remotely sensed images. Notice (see, for example, (f)) how errors occur due to the Viterbi search procedure not detecting the appropriate features in the queue and so skipping to the correct state on another building. 


\section{Conclusions}

In this paper we have investigated some new extensions and applications of HMMs to shape boundary generation. In this case "shape" has been defined as a sequence of SSs which experts use to depict critical properties of objects. Results are encouraging though require extensions to the normal application of the Viterbi method. The specific HMM investigated here, the What-HMM, offers a somewhat different definition of sets of equivalent "shapes": those whose sequences of $\mathrm{p}(\mathrm{SS}) \mathrm{p}(\mathrm{FS} / \mathrm{SS})$ products are identical. In the cases studied here, this produces classes of equivalent right-angles polyhedral objects with identical local shape state transitions given specific types of observed features.

\section{References}

1. C. Bregler. Learning and recognizing human dynamics in video sequences. In Proceedings of the IEEE Computer Vision and Pattern Recognition, pages 1-8, 1997.

2. A. Jain and R. Dubes, editors. Algorithms for Clustering Data. Prentice Hall, Englewood Cliffs, NJ, 1988.

3. L. Rabiner. A tutorial on hidden markov models and selected applications in speech recognition. Proceedings of the IEEE, 77(2):257-286, 1989.

4. R. Rao and D. Ballard. Learning saccadic eye movements using multiscale spatial filters. In G. Tesauro, D. Touretzky, and T. Leen, editors, Advances in Neural Information Processing Systems 7, pages 893-900. MIT Press, 1995.

5. R. Rimey and C. Brown. Selective attention as sequential behavior: Modeling eye movement with an augmented hidden markov model. University of Rochester Computer Science Technical Report, 327, 1990.

6. I. Rybak, V. Gusakova, A. Golovan, L. Podladchikova, and N. Shevtsova. A model of attention-guided visual perception and recognition. Vision Research, 38:2387-2400, 1998.

7. E. Simoncelli and H. Farid. Steerable wedge filters for local orientation analysis. IEEE Transactions on Image Processing, 5(9):1377-1381, 1995. 